\title{
Ras signaling regulates osteoprogenitor cell proliferation and bone formation
}

\author{
Garyfallia Papaioannou', Fatemeh Mirzamohammadi ${ }^{1}$ and Tatsuya Kobayashi' ${ }^{*, 1}$
}

During endochondral bone development, osteoblasts are continuously differentiated from locally residing progenitor cells. However, the regulation of such endogenous osteoprogenitor cells is still poorly understood mainly due to the difficulty in identifying such cells in vivo. In this paper, we genetically labeled different cell populations of the osteoblast linage using stagespecific, tamoxifen-inducible Cre transgenic mice to investigate their responses to a proliferative stimulus. We have found that overactivation of Kras signaling in type II collagen-positive, immature osteoprogenitor cells, but not in mature osteoblasts, substantially increases the number of their descendant stromal cells and mature osteoblasts, and subsequently increases bone mass. This effect was mediated by both, the extracellular signal-regulated kinase (ERK) and phosphoinositide 3 kinase (PI3K), pathways. Thus we demonstrate that Ras signaling stimulates proliferation of immature osteoprogenitor cells to increase the number of their osteoblastic descendants in a cell-autonomous fashion.

Cell Death and Disease (2016) 7, e2405; doi:10.1038/cddis.2016.314; published online 13 October 2016

Bones are formed and maintained by multiple types of bone cells. ${ }^{1}$ Mineralized bone matrix is produced by osteoblasts. Osteoblasts produce a large amount of bone matrix proteins including type I collagen and osteocalcin. Osteoblasts then die or further differentiate into osteocytes that are embedded in the bone matrix. ${ }^{2,3}$ Bone is constantly remodeled throughout life. During this process, osteoblasts are continuously differentiated from locally residing mesenchymal progenitor cells. Thus, proliferation and differentiation of the osteoblast progenitors need to be well coordinated to maintain adequate bone mass and function.

However, characteristics, kinetics and regulation of such endogenous osteoprogenitor cells are still poorly understood, primarily due to the lack of proper tools for in vivo analysis of such cells.

Recently, it has been shown that a subset of early osteoprogenitor cells show a promoter activity of the type II collagen gene (Col2a1); a lineage tracing study using Col2-creER and Cre reporter transgenic mice in which tamoxifen injection genetically labels Col2-positive cells shows that Col2-positive cells include self-renewing early osteoprogenitor cells. ${ }^{4}$ Upon lineage commitment, osteoprogenitor cells start expressing Sp7 (Osterix; Osx). ${ }^{3-5}$ In contrast, mature osteoblasts and their descendants can be exclusively labeled by the use of short promoter fragments of the type I collagen gene (Col1a1). ${ }^{3,6}$

In this study, using stage-specific, tamoxifen-inducible Cre transgenic lines, we demonstrate that Ras signaling regulates proliferation of Col2-positive osteoprogenitor cells and controls bone mass, whereas Ras overactivation shows little effect in mature osteoblasts. Thus, the difference of responsiveness to Ras signaling discriminates differentiation stages of cells of the osteoblast lineage.

\section{Results}

Col2-positive cells are self-renewing osteoprogenitors. To determine the fate of osteoblast-lineage cells in different differentiation stages, we genetically labeled cells using stage-specific, tamoxifen-inducible Cre transgenic mice and R26R-tdTomato Cre reporter mice. We crossed R26RtdTomato reporter mice ${ }^{7}$ with Cre transgenic mice expressing tamoxifen-inducible Cre recombinase driven by a type II collagen (Col2a1) promoter (Col2-creER), ${ }^{8}$ an osterix (Sp7; Osx) promoter (Osx-creER), ${ }^{9}$ or a $3.2 \mathrm{~kb}$-long type I collagen promoter (Col1-creER) ${ }^{9}$ (Figure 1a). We injected a single dose of tamoxifen into pregnant mothers at E18.5 to label Cre-expressing cells. Tamoxifen induces Cre recombination and subsequently tomato expression, and thus Cre-expressing cells as of tamoxifen injection and their descendants will express tomato red fluorescent protein.

As recently shown in postnatal mice, ${ }^{4}$ a small number of Col2-creER-expressing (Col2-positive) cells were labeled in the bone marrow 2 days after tamoxifen injection (Figures $1 \mathrm{~b}$ and e, Supplementary Figures S1A, S1D). Over time, these cells produced a significant number of descendants that differentiated into bone stromal cells, osteoblasts

\footnotetext{
${ }^{1}$ Massachusetts General Hospital and Harvard Medical School, Boston, MA, USA

*Corresponding author: T Kobayashi, Medicine, Massachusetts General Hospital and Harvard Medical School, Thier 1101, 50 Blossom Street, Boston, MA 02114, USA. Tel: +1 617726 3967; Fax: +1 617726 7543; E-mail: Kobayash@helix.mgh.harvard.edu

Abbreviations: ERK, extracellular signal-regulated kinase; p-ERK, phosphorylated extracellular signal-regulated kinase; MAPK, mitogen-activated protein kinases; MEK, MAPK/ERK kinase; PI3K, phosphoinositide 3 kinase; GTP, guanosine triphosphate; TRAP, tartrate-resistant acid phosphatase; $\mu$ CT, microcomputed tomography; H/E, hematoxylin eosin staining; TUNEL, terminal deoxynucleotidyl transferase (TdT) dUTP nick-end labeling; RTK, receptor tyrosine kinase; Ral GEF, Ras-like small GTPases; FGF, fibroblast growth factor; EGF, epidermal growth factor; IGF, insulin growth factor; IACUC, institutional animal care and use committee; EDTA, ethylenediaminetetraacetic acid; BrdU, Bromodeoxyuridine; DAPI, 4',6-diamidino-2-phenylindole; ISH, in situ hybridization; S.E.M., standard error of the mean Received 06.6.16; revised 02.9.16; accepted 07.9.16; Edited by M Agostini
} 
and osteocytes (Figures 1c,d and f-h, Supplementary Figures S1B, S1C, S1E, S1F).

As expected, Osx-creER-expressing (Osx-positive) and Col1-creER-expressing (Col1-positive) cells were abundantly present on bone surface at early time points after tamoxifen injection. In these models, numbers and localization patterns of RFP-labeled cells appeared relatively constant during the 1-month chase period (Figures 1i-n, Supplementary Figures
S1G-S1N). Col1-creER-expressing cells and their descendants are mainly osteoblasts and osteocytes (Figure 1n, Supplementary Figure $\mathrm{S} 1 \mathrm{~N}$ ), whereas Osx-creER labels stromal cells, osteoblasts and osteocytes (Figure 1k, Supplementary Figure S1J). Tamoxifen injection into Osx-creER at E18.5 does not label cells in the secondary ossification center (Supplementary Figure S1H). Because the secondary ossification center is not yet formed at E18.5, this finding suggests

a

$\begin{array}{lll} & & \text { Col2-creER } \\ & \text { or } \\ \text { Osx-creER } & \text { Os } \\ \text { R26R'omato } & & \text { or } \\ \text { mouse } & X \quad & \text { Col1-creER }\end{array}$

Tamoxifen injection

mouse

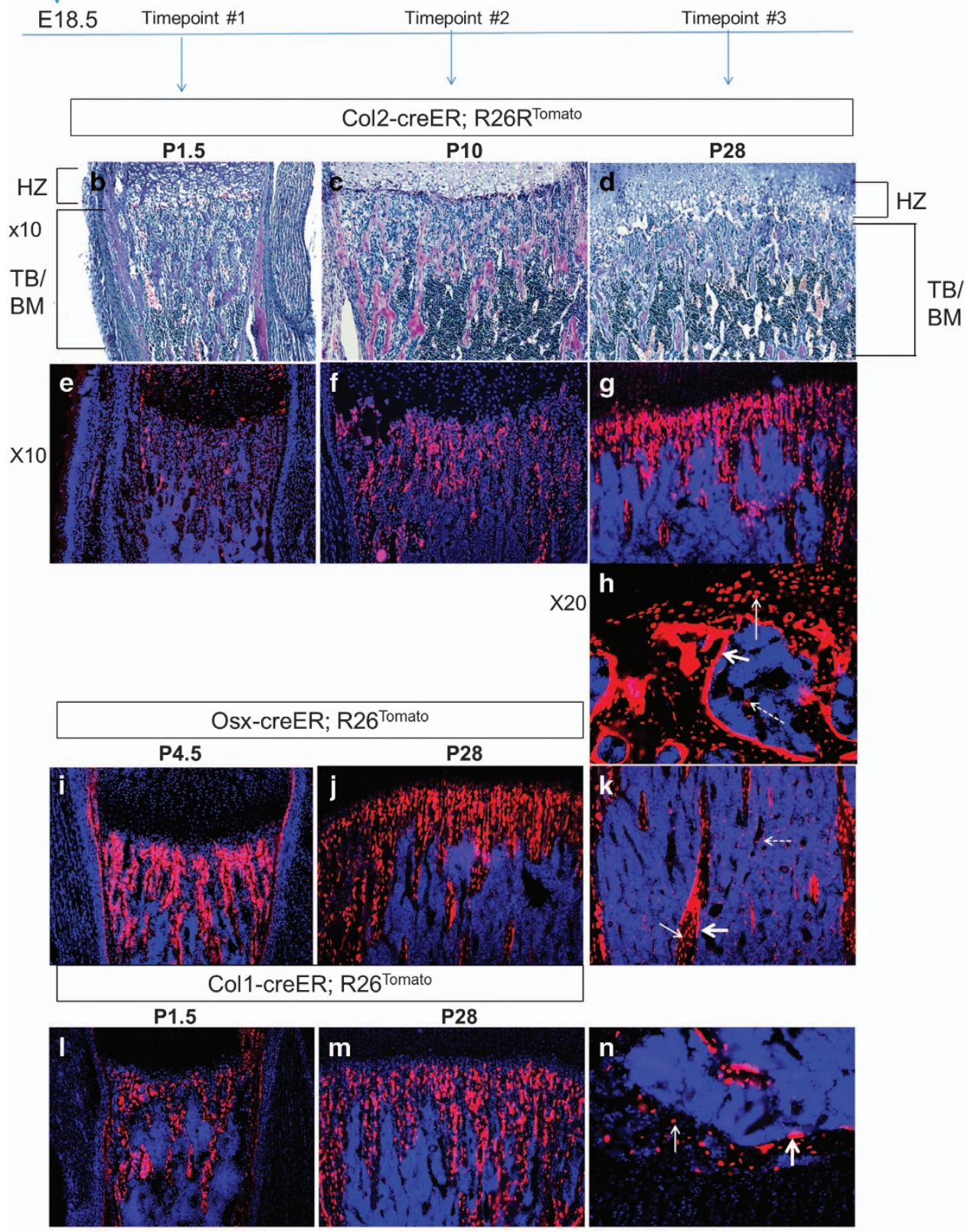


that Osx-positive cells and their descendants in the primary ossification center unlikely migrate into the secondary ossification center to serve as a source of osteoblasts.

Expression of an oncogenic Kras in Col2-positive cells increases the number of their descendant cells and trabecular bone mass. Mechanisms that regulate proliferation, differentiation and maintenance of endogenous osteoprogenitor cells in bone are still largely unknown. The recent finding that a self-renewing endogenous osteoprogenitor population can be genetically labeled using Col2-creER and R26R-tdTomato transgenic mice provided an opportunity to investigate the regulation of osteoprogenitor cells in vivo.

One of major regulators of cell proliferation is the Ras signaling pathway. The small GTPase, Ras, mediates the extracellular proliferative stimuli to downstream intracellular signaling molecules, including the extracellular signalregulated kinase (ERK) and phosphoinositide 3 kinase (PI3K) pathways, to stimulate cell cycle progression. Thus, we hypothesized that Ras-mediated signaling pathways might regulate osteoprogenitor cell proliferation.

In order to study the effect of overactivation of Ras signaling in osteoprogenitor cells in vivo, we expressed an active form of Kras (Kras $\left.{ }^{\mathrm{G} 12 \mathrm{D}}\right)^{10}$ in Col2-positive cells that were simultaneously labeled with Tomato red fluorescent protein using Col2-creER transgenic mice. Expression of Kras ${ }^{\mathrm{G} 12 \mathrm{D}}$ and Tomato was induced at E18.5 by a single dose of tamoxifen injection into pregnant mothers.

Two days after tamoxifen injection, only small numbers of Tomato-expressing cells were found in the metaphyseal region of both $\mathrm{Kras}^{\mathrm{G} 12 \mathrm{D}}$-positive and negative mice (Figures 2a-d). However, 1 month later Tomato-positive cells were significantly more abundant in $\mathrm{Kras}^{\mathrm{G} 12 \mathrm{D}}$-positive mice than in $\mathrm{Kras}^{\mathrm{G} 12 \mathrm{D}}$-negative mice (Figures $2 \mathrm{e}$ and $\mathrm{f}$ ). The increase in Tomato-positive cells in $\mathrm{Kras}^{\mathrm{G} 12 \mathrm{D}}$-positive mice involved osteocytes, osteoblasts as well as stromal cells (Figures $2 \mathrm{~g}$ and $\mathrm{h}$ ). Hematoxylin-eosin (H/E) staining of tibia sections showed a striking increase in trabecular bone mass in mice with Kras activation in Col2-positive cells and their descendants (Figures 2i and j). Littermates lacking Cre or Kras were used as controls, and they received the same amount of tamoxifen as the mutant mice. Kras ${ }^{\mathrm{G} 12 \mathrm{D}}$ mice without Cre recombination had the same bone phenotype as wild-type mice. In the absence of tamoxifen there were no overt bone phenotypes in mutant mice (Supplementary Figures S2A-S2B).
A similar phenotype was observed in the humerus as in the tibia upon Kras activation in Col2-postive cells (Supplementary Figures S2C-S2D). Microcomputed tomography $(\mu \mathrm{CT})$ analysis showed that there was a significant increase in the bone volume fraction, trabecular number and trabecular thickness and a decrease in trabecular separation (Figures 2k-p). The cortical bone parameters were unchanged. There were no overt changes in tartrate-resistant acid phosphatase (TRAP) staining (Supplementary Figures S2I-S2J), suggesting that bone-resorbing osteoclasts were not affected.

In addition to the increased bone mass, $\mathrm{H} / \mathrm{E}$ staining revealed a substantial increase in the number of stromal cells between the trabeculae (Figures $3 a$ and b, Supplementary Figures S2E, S2F, and Figure $4 \mathrm{~m}$ ). These stromal cells weakly expressed Col1a1 while they were negative for the mature osteoblast marker, Bglap that encodes osteocalcin (Figures 3c-f).

As expected, Kras ${ }^{\mathrm{G} 12 \mathrm{D}}$ expression increased the number of cells that are positive for phosphorylated ERK1/2 ( $p-E R K)$ over time (Figures 3g-j). The PI3K pathway, another important pathway downstream of Kras, was also found activated in the stromal cells of Kras ${ }^{\mathrm{G} 12 \mathrm{D}}$ mice, as demonstrated by the increase in phosphorylation of Akt (p-Akt) (Figures 3k and I).

To assess the proliferation of stromal cells, BrdU assay was performed. As expected, the BrdU labeling index of the stromal cell population in the Kras ${ }^{\mathrm{G} 12 \mathrm{D}}$ mouse was increased at postnatal day P10 (Figures 3m-q).

Overactivation of Ras signaling influences cell survival. In order to test whether oncogenic Ras expression affects cell survival, a cell death assay (TUNEL assay) was performed to compare the apoptosis rate in the primary spongiosa of Kras $^{\text {G12D }}$ mice and wild-type mice (Supplementary Figures S3E-S3G). Although a tendency for increased apoptosis in $\mathrm{Kras}^{\mathrm{G} 12 \mathrm{D}}$ mice was observed, the difference in cell death signals counted was not statistically significant (control versus mutant: $11 \pm 4.6$ versus $19.7 \pm 4 P=0.07, n=3$ each). This suggests that the increase in proliferation of stromal cells is the dominant mechanism for bone increase in the Kras $^{\mathrm{G} 12 \mathrm{D}}$ mice.

We then wanted to address the question whether the effect of Kras overexpression in Col2-positive cells was age-specific. Activating Kras at P20 in Col2-positive cells caused a similar increase in bone and stromal cells, suggesting that Col2-positive cells are continuously present and serve as

Figure 1 The fate and localization of bone-forming cells: Col2-, Osx- and Col1-positive cells during bone development (see also Supplementary Figure S1). (a) Experimental design. R26R-tdTomato reporter mice were crossed with transgenic mice expressing creER under the control of Col1, Osx and Col2 promoters. A single dose of tamoxifen $(0.1 \mathrm{mg} / \mathrm{g})$ was intraperitoneally (i.p.) injected into pregnant mothers at E18.5. (b-d) Hematoxylin/eosin-stained paraffin sections of the proximal metaphysis of the tibia at indicated ages $(\mathrm{P}=$ postnatal day, $\mathrm{TB}=$ trabecular bone, $\mathrm{BM}=$ bone marrow, $\mathrm{HZ}=$ hypertrophic chondrocyte zone). (e-g) Fluorescent images of the tibia of $\mathrm{B}-\mathrm{D}(\times 10)$. Blue:Dapi; Red: Tomato red fluorescent protein. (h) A cryosection ( $\times 20$ magnification) of the secondary ossification center in the Col2-creER;R26R ${ }^{\text {tomato }}$ mouse tibia at P28. Tomato-labeled cells are observed on the bone surface (osteoblasts; thick arrow), inside of the bone matrix (osteocytes; thin arrow), and in the bone marrow (dotted arrow). (i-j) Cryosections ( $\times 10$ magnification) of the Osx-creER;R26R ${ }^{\text {tomato }}$ mouse tibiae at indicated ages. Blue, DAPI; red, Tomato red fluorescent protein. (k) Cryosection ( $\times 10$ magnification) of the OsxcreER;R26R ${ }^{\text {tomato }}$ mouse tibia bone marrow at P28. Blue: DAPI; red: Tomato red fluorescent protein; thick arrow: osteoblasts; thin arrow: osteocytes; dotted arrow: stromal cells. (I-m) Cryosections ( $\times 10$ magnification) of the Col1-creER;R26R ${ }^{\text {tomato }}$ mouse tibia pulsed with tamoxifen at age E18.5. Tomato (red fluorescent protein) labeled the Col1-positive cells at age E18.5 and cell fate was chased at ages P1.5 and P28. (n) Cryosection of Col1-creER;R26R tomato mouse tibia showing the secondary ossification center (×20 magnification) at P28. Blue, DAPI; red, Tomato red fluorescent protein. thin arrow: osteocytes; thick arrow: osteoblasts. No tomato-positive cells were found in the bone marrow. $n=5$ mice per group 

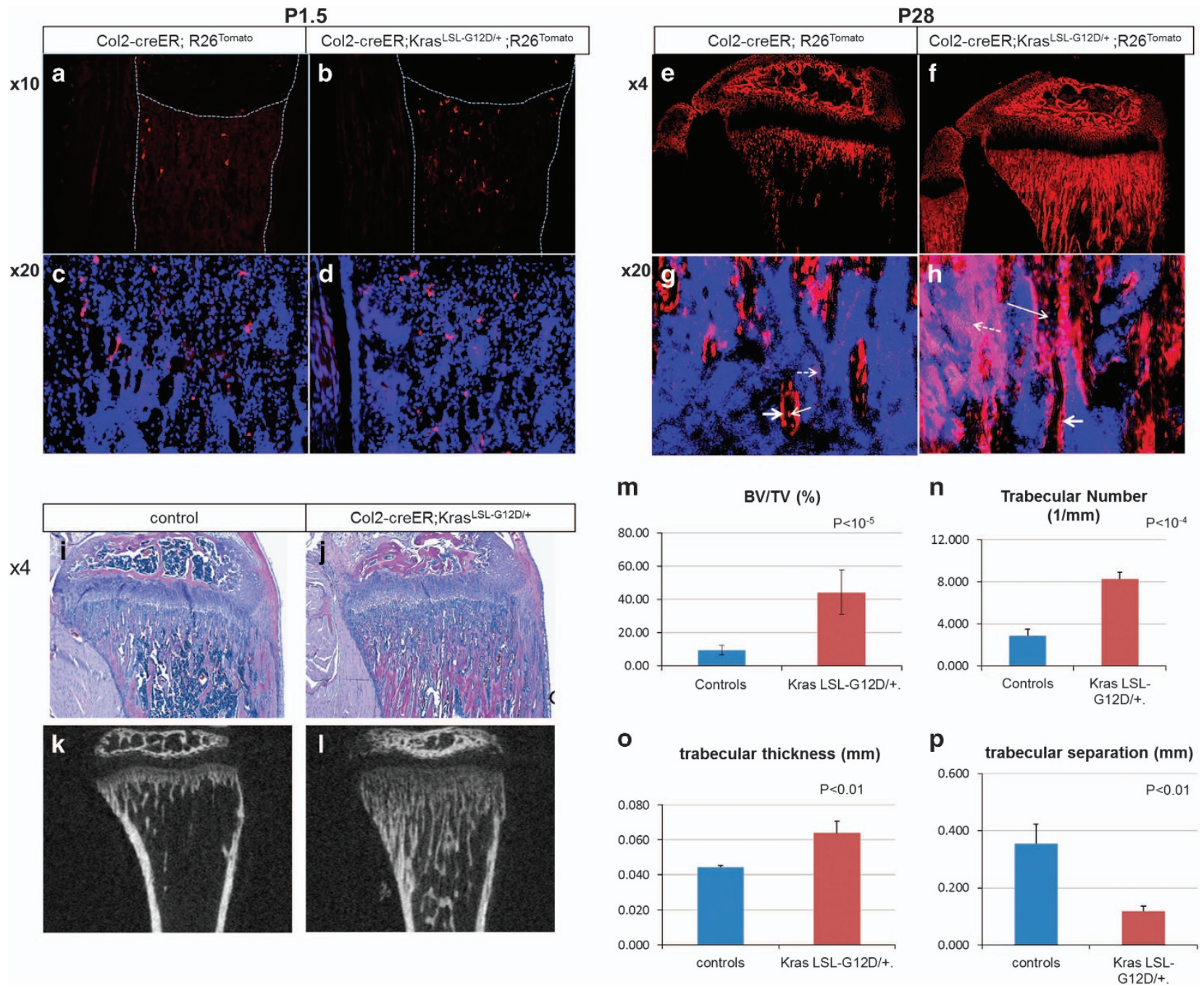

Figure 2 Expression of $\mathrm{Kras}^{\mathrm{G} 12 \mathrm{D}}$ in Col2-positive cells at E18.5 increases the number of their descendants and the trabecular bone mass (see also Supplementary figure S2). (a-d) Tomato-positive cells in the tibial metaphysis of Col2-creER;R26R ${ }^{\text {tomato }}$ mice with wild-type Kras (a, c) and Kras ${ }^{\mathrm{G} 12 \mathrm{D}}$ (b,d) at P1.5. Mice were treated with tamoxifen at E18.5. A,B: $\times 4$ magnification. c,d: $\times 20$ magnification. Blue, DAPI; red, Tomato red fluorescent protein. Dotted lines indicate the borders of tibia. The middle dotted line indicates the border between growth plate and primary spongiosa (trabecular bone). Magnifications of primary spongiosa of $\mathbf{a}$ and $\mathbf{b}$ are shown in $\mathbf{c}$ and $\mathbf{d}$ respectively $(n=3)$.

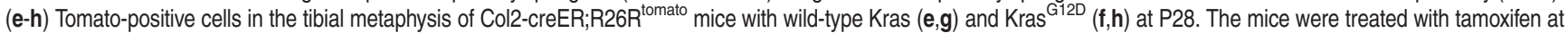
E18.5. E,F: $\times 4$ magnification. g,h: $\times 20$ magnification. Blue, DAPI; red, Tomato red fluorescent protein; arrow: osteocytes; double arrow: osteoblasts; dotted arrow: stromal cells $(n=4)$. (i-j) Hematoxylin/eosin-stained paraffin sections of tibias at P28 from Col2-creER mice with wild-type Kras (i) and Kras ${ }^{\mathrm{G} 12 \mathrm{D}}$ (j) after Kras activation at E18.5 ( $\times 4$ magnification). Mice harboring Kras ${ }^{\mathrm{G} 12 \mathrm{D}}$ show increased trabecular bone with a minimal effect in the growth plate structure $(n=8)$. (k-I) MicroCT pictures of tibias at P28 from Col2-creER mice with wild-type Kras (k) and Kras ${ }^{G 12 D}(\mathbf{I})$ after Kras activation at E18.5. (m-p) Graphs showing comparisons of bone volume fraction ( $\left.\mathbf{m}\right)$, trabecular number (n), trabecular thickness $(\mathbf{o})$ and trabecular separation (p) measured by microCT in mice with wild-type Kras or Kras ${ }^{\mathrm{G} 12 \mathrm{D}}$ at P28 $(n=3$ and $n=4$ for mutant and control groups, respectively)

osteoprogenitor cells in postnatal bone (Supplementary Figures S3A-S3D).

MAPK and PI3K are the main signaling pathways responsible for the increase in bone with Kras activation. Kras signals mainly through the mitogen-activated protein kinase (MAPK) and PI3K pathways. ${ }^{11}$ In order to investigate whether activation of these signaling pathways was responsible for the bone phenotype of Kras mice, we pharmacologically inhibited the ERK and PI3K pathways. We injected U0126, an inhibitor of ERK kinase (MEK), or LY294002, a PI3K inhibitor from E18.5 through P21. Both
MEK inhibition (Figures $4 \mathrm{~h}$ and $\mathrm{k}$ ) and PI3K inhibition (Figures $4 \mathrm{i}$ and I) partially, but significantly, rescued the phenotype in Col2-creER; Kras ${ }^{\mathrm{G} 12 \mathrm{D}}$ mice injected with tamoxifen at E18.5, as demonstrated by the decreased stromal cell number and trabecular bone (Figure 4h, i, k, I, m and comparison with figures $4 \mathrm{~g}, \mathrm{j}$ ). In both MEK inhibition and PI3K inhibition, their downstream molecules ( $p$-ERK and p-Akt signals respectively) were suppressed after the treatment with inhibitors (Supplementary Figures S4A-S4F). These findings suggest that activation of both MAPK and PI3K pathways contribute to the bone phenotype of Kras $^{\mathrm{G} 12 \mathrm{D}}$ mice. 
Kras $^{G 12 D}$ expression in Col1-positive cells does not increase stromal cells or bone mass. Next, we investigated the effect of Kras activation in lineage-committed osteoblasts. We used Osx-creER and Col1-creER to target

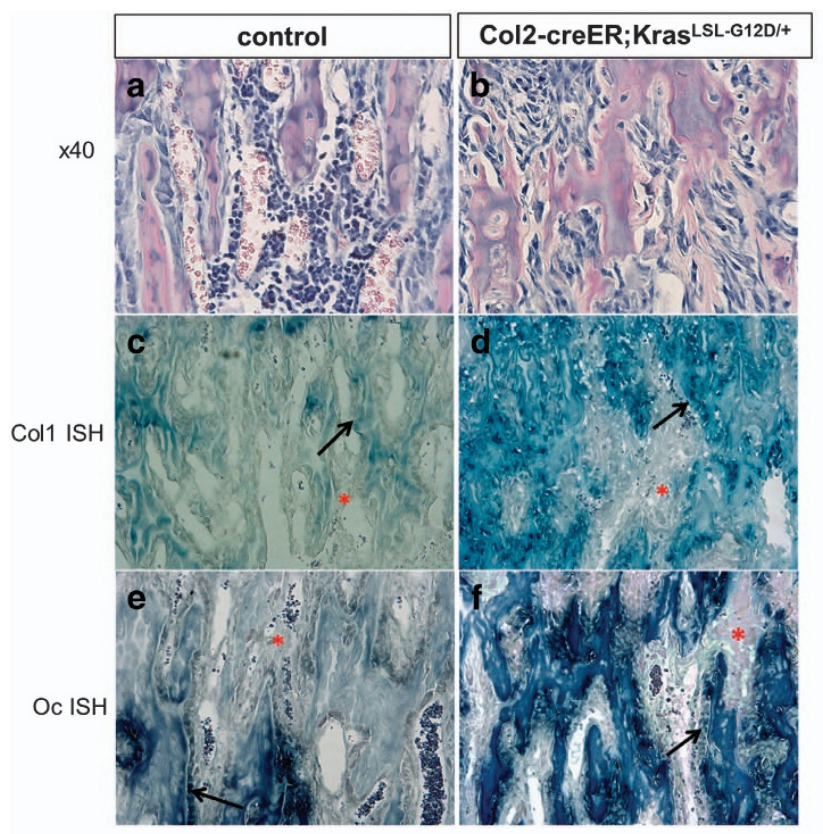

osteoblasts in early and late differentiation stages, respectively. A single dose of tamoxifen was injected into mice at E18.5 to induce expression of $\mathrm{Kras}^{\mathrm{G12D}}$ and Tomato in Osx-positive or Col1-positive cells.
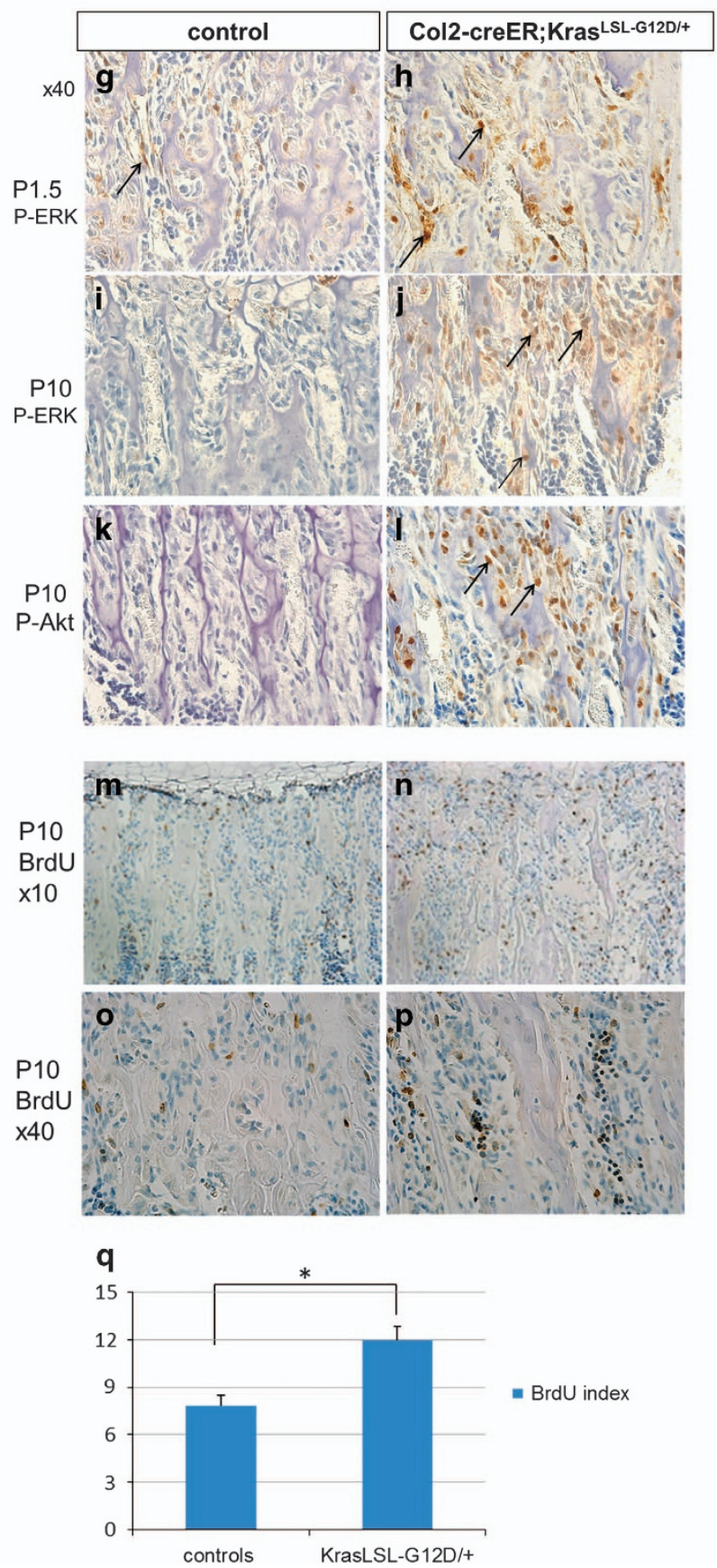

Figure 3 Activation of Kras oncogene in Col2 cells at perinatal age increases stromal cell numbers (see also Supplementary figure S3). (a-b) Hematoxylin/eosin-stained paraffin sections showing the stromal cells between the trabeculae in P28 mice (secondary spongiosa) control (a) and Kras ${ }^{\mathrm{G} 12 \mathrm{D}}$ (b) after Kras activation at E18.5. $\times 40$ magnification. (c-f) In situ hybridization for collagen 1 (Col1 ISH) (c,d) and osteocalcin (Oc ISH) (e,f) in the secondary spongiosa of P28-old mice control $(\mathbf{c}, \mathbf{e})$ or Kras ${ }^{\mathrm{G} 12 \mathrm{D}}$ (d,f) after tamoxifen injection at E18.5. Stromal cells are not stained for these markers. Black arrows, osteoblasts; red asterisks, stromal cells. (g-j) Immunohistochemistry for p-ERK at P1.5 (g, h) and P10 (i,j) in the tibia secondary spongiosa of control $(\mathbf{g}, \mathbf{i})$ and $\mathrm{Kras}^{\mathrm{G} 12 \mathrm{D}}(\mathbf{h}, \mathbf{j})$ after tamoxifen injection at E18.5. Arrows show representative cells stained with anti- p-ERK. (k-I) Immunohistochemistry for p-Akt at P10 in the tibia secondary spongiosa of control (k) or Kras ${ }^{\mathrm{G} 12 \mathrm{D}}$ (I) after tamoxifen injection at E18.5. Arrows show representative cells stained with anti- $p$-Akt antibody. $(\mathbf{m}-\mathbf{p})$ BrdU (Bromodeoxyuridine) labeling of proliferating cells in the metaphysis of mice (tibia) control ( $m, 0)$ and $\mathrm{Kras}{ }^{\mathrm{G} 12 \mathrm{D}}$ $(\mathbf{n}, \mathbf{p})$ at P10 after Kras activation at E18.5. BrdU-positive cells are stained brown. (q) The BrdU index, calculated as the percentage of BrdU-labeled stromal cells, was significantly increased upon $\mathrm{Kras}^{\mathrm{G} 12 \mathrm{D}}$ activation. Stromal cells were defined as the non-hematopoietic cells that were not attached to the bone matrix. Data are represented as mean \pm S.E.M.; $n=3,{ }^{*} P=0.002$ 

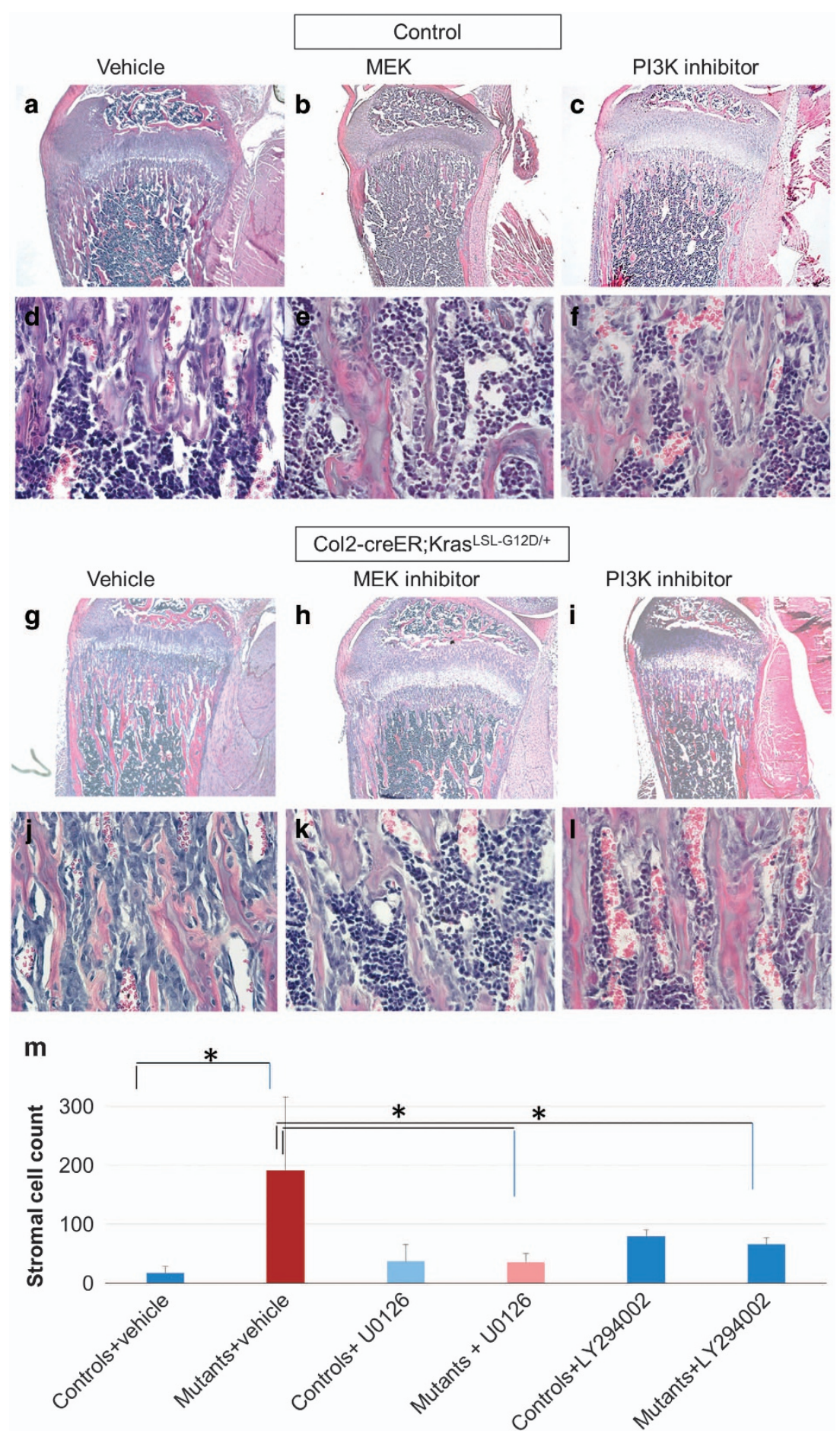

Figure 4 The MAPK and PI3K pathways are responsible for the increase in bone. (a-f) Hematoxylin/eosin-stained paraffin sections of tibias from Col2-creER or Kras ${ }^{\mathrm{G} 12 \mathrm{D}}$ (control) mice at P21 (tamoxifen injection at E18.5) treated with vehicle (methylcellulose) (a,d), MEK inhibitor (U0126; $5 \mathrm{mg} / \mathrm{kg})(\mathbf{b}, \mathbf{e})$, or Pl3K inhibitor (LY294002 100 mg/kg) (c,f). $(\mathbf{g}-\mathbf{l})$ Hematoxylin/eosin-stained paraffin sections of tibias from Col2-creER;Kras ${ }^{\text {LLL-G12D/+ }}$ mice at P21 after tamoxifen injection at E18.5 (g,j), with MEK inhibitor U0126 injections $(\mathbf{h}, \mathbf{k})$ or PI3K inhibitor LY294002 injections (i,I). After MEK or PI3K inhibition, mice harboring Kras ${ }^{\mathrm{G} 12 \mathrm{D}}$ show reduced trabecular bone and significantly smaller number of stromal cells than the vehicle-injected mutants. a,b,c, $\mathbf{g}, \mathbf{h}, \mathbf{i}: \times 4$ magnification. $\mathbf{d}, \mathbf{e}, \mathbf{f , j ,}, \mathbf{k}, \mathbf{l}: \times 40$ magnification. $(\mathbf{m})$ Stromal cell counts in control and mutant mice injected with vehicle or MEK inhibitor or PI3K inhibitor. Data are represented as mean \pm S.E.M.; $n=3,{ }^{\star} P<0.05$. Spindle-shaped cells, not attached to the bone matrix were counted in the center of secondary spongiosa in $\times 20$ magnification 

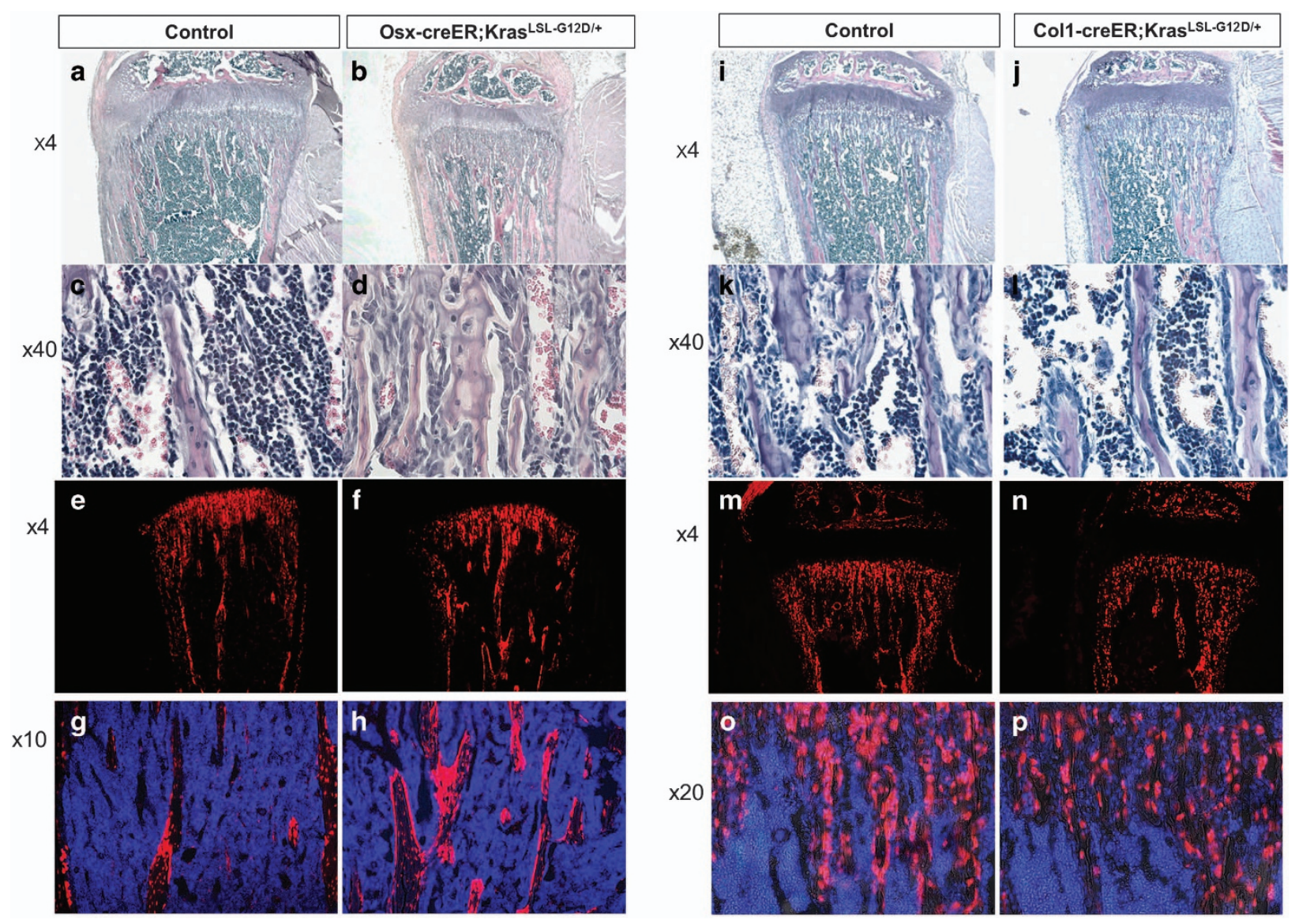

Figure 5 Overexpression of Kras in Osx cells perinatally causes a milder increase in bone and stromal cells, while in Col1 cells it has no effect (see also Supplementary figure S4). (a-b) Hematoxylin/eosin-stained paraffin sections of tibias from P28-old Osx-creER mice control $(\mathbf{a}, \mathbf{c})$ and Kras ${ }^{\mathrm{G} 12 \mathrm{D}}$ (b,d) after Kras activation at E18.5. a,b: $\times 4$ magnification, c,d: $\times 40$ magnification. (e-h) Cryosections showing tomato-positive cells in P28-old Osx-creER mice control (e,g) and Kras ${ }^{\mathrm{G} 12 \mathrm{D}}$ (f,h) after tamoxifen injection at E18.5. e,f $\times 4$ magnification; $\mathbf{g}, \mathbf{h} \times 10$ magnification. Blue, DAPI; red, Tomato red fluorescent protein. (i-I) Hematoxylin/eosin-stained paraffin sections of tibias from P28 Col1-creER mice control (i,k) and Kras ${ }^{G 12 D}$ (j,I) after tamoxifen injection at E18.5. i,j: $\times 4$ magnification, $\mathbf{k}, \mathbf{l}: \times 40$ magnification. $(\mathbf{m}-\mathbf{p})$ Cryosections showing tomato-positive cells in P28 Col1-creER mice with wild-type $\operatorname{Kras}(\mathbf{m}, \mathbf{0})$ and $\operatorname{Kras}^{\mathrm{G} 12 \mathrm{D}}(\mathbf{n}, \mathbf{p})$ after tamoxifen injection at E18.5. $\mathbf{m}, \mathbf{n}: \times 4$ magnification, $\mathbf{0}, \mathbf{p}: \times 20$ magnification. Blue, DAPI; red, Tomato red fluorescent protein. For both the Osx model and Col1 model $n=5$ mice per group

Kras $^{\mathrm{G} 12 \mathrm{D}}$ expression in Osx-positive cells caused a phenotype similar to, but somewhat milder than, that of the Col2creER model (Figures 5a-d). Tomato-positive Osx-positive cells and their descendants were found in both bone and stromal compartments (Figures 5e-h).

In contrast, activating Kras in the Col1-positive cells caused no significant changes in bone or stromal cells at P28 (Figures 5i-I), despite that Kras ${ }^{\mathrm{G} 12 \mathrm{D}}$ expression significantly increased ERK phosphorylation in osteoblasts (Supplementary Figures S5A-S5D). Consistent with this result, there was no significant increase in tomato-positive Col1-positive cell descendants (Figures $5 \mathrm{~m}$-p). These results suggest that Ras activation has no stimulatory effect in differentiated osteoblasts.

\section{Discussion}

In this study, we investigated the role of Ras signaling in three different cell populations of the osteoblast lineage, that is, Col2-, Osx- and Col1-positive cells, during bone development.
Kras overactivation in Col2-positive or Osx-positive cells in bone at E18.5 led to a significant increase in number of their descendant cells and trabecular bone mass. In contrast, Kras overactivation in Col1-positive cells did not show significant effects in cell number or bone mass, suggesting that Col2- and Osx-positive cells, but not Col1-positive cells, include proliferative osteoblast progenitors. This study also demonstrates that Ras signaling is an important positive regulator of proliferation of early osteoprogenitor cells.

Multiple signaling molecules, including receptor tyrosine kinases (RTKs), ${ }^{12}$ have been implicated in regulation of proliferation and survival of bone progenitor cells. ${ }^{13,14}$ Kras, a small GTPase activated by RTKs, triggers downstream effector pathways, such as the MAPK (mitogen-activating protein kinase), PI3K (Phosphatidylinositol 3'-kinase) and Ral-GEF (Ras-like small GTPases) pathways, to regulate cellular functions including proliferation, differentiation and apoptosis. ${ }^{15}$ Multiple Rasactivating molecules, including fibroblast growth factors (FGFs)/ FGF receptors ${ }^{16}$ epidermal growth factors (EGFs)/ EGF receptors $^{17}$ and insulin-like growth factors (IGFs) and their receptors, ${ }^{18}$ have been implicated in regulating bone development. 
Ras signaling has been shown to increase stem/progenitor cell proliferation in multiple tissues. Kras activation has been shown to increase proliferation of bone marrow hematopoietic cells. ${ }^{19-21}$ Additionally an oncogenic Kras increases proliferation of mouse embryonic fibroblasts ${ }^{22}$ and facilitates selfrenewal of a subtype of alveolar stem cells. ${ }^{23}$ Additionally Ras activation by partial loss of $\mathrm{Nf1}$ gene in osteoprogenitor cells was found to increase proliferation and cause premature apoptosis. ${ }^{24}$ In the present model apoptosis was not found significantly increased; thus, we conclude that the increase in proliferation is the dominant effect of Kras activation in osteoprogenitor cells.

However, there have been relatively limited studies that investigate the role of Ras-MAPK signaling pathway in osteoblast progenitor cells in bone in vivo. It has been shown that the ERK signaling pathway, a major downstream mediator of Ras, in mesenchymal progenitor cells promotes osteoblast differentiation and bone formation during early skeletal development. ${ }^{25}$ Nonetheless, the proliferative effect of ERK signaling specifically in osteoprogenitor cells has not been addressed. In another study in which a dominant negative or a constitutively active MEK1 was expressed in relatively mature osteoblasts, ERK signaling stimulated osteoblast differentiation without obvious changes in proliferation. ${ }^{26}$ The result of this study is consistent with our finding that Kras overactivation in Col1-positive mature osteoblasts had little effect in bone mass or stromal cell proliferation. In our study, we found that ERK inhibition significantly reduced the number of stromal cells in Kras ${ }^{\mathrm{G} 12 \mathrm{D}}$ mice, suggesting that ERK overactivation is, at least in part, responsible for the proliferative effect of $\mathrm{Kras}^{\mathrm{G} 12 \mathrm{D}}$ expression in Col2-positive osteoprogenitors. We also found that PI3K inhibition partially reduced stromal cells and partially rescued the bone phenotype caused by Kras ${ }^{\mathrm{G} 12 \mathrm{D}}$ expression. Thus our study demonstrates that in addition to the ERK pathway, the PI3K pathway also plays an important role in osteoprogenitor proliferation.

To investigate the pathways downstream of Kras we injected mice with small molecule inhibitors of MEK and PI3K. With the dosage and timeframe of these experiments we did not observe significant changes in the mouse body size or condition. The wild-type control mice injected with MEK inhibitor showed no appreciable changes in bone.

Over-expression of active Kras in Col2-Cre-positive cells is not physiologically present during development, and therefore our findings do not necessarily demonstrate that endogenous Ras signaling is a physiologic regulator of osteoprogenitor cells. Nevertheless, using this system, we are able to show that Col2-positive or Osx-positive cells, but not Col1-positive cells include osteoprogenitor cells that can be discriminated by responses to Ras signaling. Additionally these experiments show that MAPK and PI3K pathways activation in the above cell populations can activate bone formation.

The loss of balance between bone formation and bone resorption results in osteoporosis. ${ }^{27}$ Since treatment options to increase bone formation are currently limited, ${ }^{28}$ novel bone anabolic therapies are highly desired. Our study shows that stimulating Ras signaling in endogenous osteoprogenitor cells can be a valid treatment strategy for osteoporosis.

\section{Materials and Methods}

Mice. Col2a1-creERT2, ${ }^{8}$ Osx-creERT2 ${ }^{3}$ Col1-creERT2, ${ }^{9}$ and Cre-dependent Kras transgenic, loxP-stop-loxP-Kras ${ }^{\mathrm{G} 12 \mathrm{D} 10}$ mice have been described elsewhere. The Cre reporter, Rosa26-loxP-stop-loxP-tdTomato mice were acquired from Jackson Laboratory (Bar Harbor, ME, USA). ${ }^{7}$ All procedures were conducted in compliance with the Guideline for the Care and Use of Laboratory Animals approved by Massachusetts General Hospital's Institutional Animal Care and Use Committee (IACUC). Mice were in a mixed genetic background. Comparisons were made between littermates. Tamoxifen injections were given i.p. at a dose of $0.1 \mathrm{mg} /$ $\mathrm{g}$ to pregnant females as described elsewhere. ${ }^{4}$

Histology. For histological analysis mice were dissected, fixed in $10 \%$ formalin overnight and decalcified in 10\% EDTA for a period ranging from 1 to 14 days, depending on the age of the mice. For H/E staining, BrdU staining, immunohistochemistry and in situ hybridization, samples were paraffin processed and cut. For fluorescent reporter analysis decalcified samples were cryoprotected in $30 \%$ sucrose/ phosphate buffer saline (PBS) solutions then in $30 \%$ sucrose/PBS:OCT (1:1) solutions, each overnight at $4^{\circ} \mathrm{C}$. Samples were embedded in OCT compound (TissueTek, Sakura, Torrance, CA, USA) and transferred to dry ice to solidify OCT. Samples were cryosectioned at the thickness of $15 \mu \mathrm{m}$ using a cryostat (Leica CM1850). Nikon Eclipse E800 microscope was used for imaging. Images were merged with Spot Advanced Software.

Immunohistochemistry and in situ hybridization. Immunohistochemistry analysis for phosphorylated ERK (phospho-ERK) was performed as previously described using the Perkin Elmer TSA biotin system kit. ${ }^{29}$ The primary antibody directed against phospho-p44/42 (ERK1/2) (Cell Signaling, Danvers, MA, USA, catalog number:\#43700) was diluted 1:300 and the secondary biotinylated anti-rabbit antibody (Vector, Burlingame, CA, USA, catalog number \#BA1000) was diluted 1:300. Anti p-Akt (Cell Signaling, catalog number \#2965) antibody was diluted 1:500. ISH for type 1 collagen and osteocalcin was performed according to published protocols. ${ }^{30}$ For all immunostaining experiments, samples from three mice per group were analyzed.

Cell proliferation assay. For BrdU labeling $50 \mu \mathrm{g}$ of BrdU per gram of body weight was intraperitoneally injected into mice $2 \mathrm{~h}$ before euthanasia. BrdU was detected using the BrdU in situ staining kit (invitrogen, Waltham, MA, USA). The BrdU labeling index was calculated as the ratio of BrdU-positive nuclei over total nuclei of stromal cells of the metaphyseal tibia.

TRAP staining. Slides were deparaffinized and rehydrated. TRAP reagent consisted of $6 \mathrm{ml} 50 \mathrm{mM}$ tatrate in acetate buffer (PH 5.0), $0.5 \mathrm{mg}$ Naphthol As-Mx, $50 \mu \mathrm{l} \mathrm{N}, \mathrm{N}$ dimethylamide and $3 \mathrm{mg}$ fast red violet LB. TRAP reagent was added for 5-10 min and once satisfactory color was observed, slides were washed with water and then with PBS. At the end the slides were mounted with water-based medium.

Cell death assay (TUNEL assay). Cell death was evaluated on sections using an in situ cell death detection kit (Roche, Branford, CT, USA) according to the manufacturer's instructions.

MicroCT analysis. A high-resolution desktop micro-tomographic imaging system ( $\mu$ CT40, Scanco Medical AG, Basserdorf, Switzerland) was used to assess trabecular and cortical in the proximal metaphysis and diaphysis of the tibia, respectively. Scans were acquired and analyzed as previously described. ${ }^{31}$ Image acquisition and analysis were performed according to the JBMR guidelines for the use of $\mu \mathrm{CT}$ in rodents. ${ }^{32}$

To assess the trabecular architecture, transverse CT slices were evaluated in a $1500 \mu \mathrm{m}$ (150 slices) region beginning $750 \mu \mathrm{m}$ below the proximal growth plate and extending distally. The trabecular bone region was identified by semi-manually tracing with the assistance of an auto-thresholding software algorithm. A mineral density threshold of $225 \mathrm{mgHA} / \mathrm{cm}^{3}$ was used for all images to segment bone from soft tissue.

Rescue experiments- inhibitors. The MEK1 inhibitor (U0126) and the PI3K inhibitor (LY294002) were purchased from Selleckchem (Houston, TX, USA). Inhibitors were first dissolved in DMSO according to the manufacturer's instructions, then diluted into PBS, and injected daily into pregnant as well as nursing mothers intraperitoneally from E18.5 through P9.5. Subsequently, the inhibitors were injected to pups. MEK inhibitor was injected at a dose of $5 \mathrm{mg} / \mathrm{kg}$ per day. PI3K inhibitor was injected at a dose of $100 \mathrm{mg} / \mathrm{kg}$ four times a week. 


\section{Conflict of Interest}

The authors declare no conflict of interest.

Acknowledgements. We thank Jacqueline Lees and Simona Nedelcu for valuable discussion and the Center for Skeletal Research Core (NIH P30 AR066261) for histological analysis. This work was supported by the National Institutes of Health (AR056645 to T.K.) and Harvard Stem Cell Institute (SG-0090-13 to T.K.).

\section{Author contributions}

G.P. and T.K. conceived the project, designed the study, and wrote the paper. G.P., F.M. and T.K. performed the experiments and analyzed the data.

1. Sims NA, Vrahnas $C$. Regulation of cortical and trabecular bone mass by communication between osteoblasts, osteocytes and osteoclasts. Arch biochem biophys 2014; 561: 22-28.

2. Marie PJ. Transcription factors controlling osteoblastogenesis. Arch biochem biophys 2008; 473: 98-105.

3. Maes C, Kobayashi T, Selig MK, Torrekens S, Roth SI, Mackem S et al. Osteoblast precursors, but not mature osteoblasts, move into developing and fractured bones along with invading blood vessels. Dev cell 2010; 19: 329-344.

4. Ono N, Ono W, Nagasawa T, Kronenberg HM. A subset of chondrogenic cells provides early mesenchymal progenitors in growing bones. Nat cell biol 2014; 16: 1157-1167.

5. Park D, Spencer JA, Koh BI, Kobayashi T, Fujisaki J, Clemens TL et al. Endogenous bone marrow MSCs are dynamic, fate-restricted participants in bone maintenance and regeneration. Cell stem cell 2012; 10: 259-272.

6. Rossert J, Eberspaecher $\mathrm{H}$, de Crombrugghe B. Separate cis-acting DNA elements of the mouse pro-alpha 1 (I) collagen promoter direct expression of reporter genes to different type I collagen-producing cells in transgenic mice. $J$ cell biol 1995; 129: 1421-1432.

7. Madisen L, Zwingman TA, Sunkin SM, Oh SW, Zariwala HA, Gu H et al. A robust and high-throughput $\mathrm{Cre}$ reporting and characterization system for the whole mouse brain. Nat neurosci 2010; 13: 133-140.

8. Nakamura E, Nguyen MT, Mackem S. Kinetics of tamoxifen-regulated Cre activity in mice using a cartilage-specific $\operatorname{CreER}(\mathrm{T})$ to assay temporal activity windows along the proximodistal limb skeleton. Dev dynam 2006; 235: 2603-2612.

9. Maes C, Kobayashi T, Kronenberg HM. A novel transgenic mouse model to study the osteoblast lineage in vivo. Ann NY Acad Sci 2007; 1116: 149-164.

10. Johnson L, Mercer K, Greenbaum D, Bronson RT, Crowley D, Tuveson DA et al. Somatic activation of the K-ras oncogene causes early onset lung cancer in mice. Nature 2001; 410 : $1111-1116$.

11. Chetty R, Govender D. Gene of the month: KRAS. J clin pathol 2013; 66: 548-550.

12. Ng F, Boucher S, Koh S, Sastry KS, Chase L, Lakshmipathy U et al. PDGF, TGF-beta, and FGF signaling is important for differentiation and growth of mesenchymal stem cells (MSCs): transcriptional profiling can identify markers and signaling pathways important in differentiation of MSCs into adipogenic, chondrogenic, and osteogenic lineages. Blood 2008; 112 : 295-307.

13. Tan SH, Senarath-Yapa K, Chung MT, Longaker MT, Wu JY, Nusse R. Wnts produced by Osterix-expressing osteolineage cells regulate their proliferation and differentiation. Proc Natl Acad Sci USA 2014; 111: E5262-E5271.

14. Chen G, Deng C, Li YP. TGF-beta and BMP signaling in osteoblast differentiation and bone formation. Int $j$ biol sci 2012; 8: 272-288.

15. Karnoub AE, Weinberg RA. Ras oncogenes: split personalities. Nat rev Mol cell biol 2008; 9 : 517-531.

16. Ornitz DM, Marie PJ. Fibroblast growth factor signaling in skeletal development and disease. Genes dev 2015; 29: 1463-1486.

17. Chandra A, Lan S, Zhu J, Siclari VA, Qin L. Epidermal growth factor receptor (EGFR) signaling promotes proliferation and survival in osteoprogenitors by increasing early growth response 2 (EGR2) expression. J biol chem 2013; 288: 20488-20498.
18. Wang Y, Bikle DD, Chang W. Autocrine and paracrine actions of IGF-I signaling in skeletal development. Bone res 2013; 1: 249-259.

19. Chan G, Gu S, Neel BG. Erk1 and Erk2 are required for maintenance of hematopoietic stem cells and adult hematopoiesis. Blood 2013; 121: 3594-3598.

20. Braun BS, Tuveson DA, Kong N, Le DT, Kogan SC, Rozmus J et al. Somatic activation of oncogenic Kras in hematopoietic cells initiates a rapidly fatal myeloproliferative disorder. Proc Natl Acad Sci USA 2004; 101: 597-602.

21. Van Meter ME, Diaz-Flores E, Archard JA, Passegue E, Irish JM, Kotecha N et al. K-RasG12D expression induces hyperproliferation and aberrant signaling in primary hematopoietic stem/progenitor cells. Blood 2007; 109: 3945-3952.

22. Guerra C, Mijimolle N, Dhawahir A, Dubus P, Barradas M, Serrano M et al. Tumor induction by an endogenous K-ras oncogene is highly dependent on cellular context. Cancer cell 2003; 4: $111-120$.

23. Desai TJ, Brownfield DG, Krasnow MA. Alveolar progenitor and stem cells in lung development, renewal and cancer. Nature 2014; 507: 190-194.

24. Yu X, Chen S, Potter OL, Murthy SM, Li J, Pulcini JM et al. Neurofibromin and its inactivation of Ras are prerequisites for osteoblast functioning. Bone 2005; 36: 793-802.

25. Matsushita T, Chan YY, Kawanami A, Balmes G, Landreth GE, Murakami S. Extracellular signal-regulated kinase 1 (ERK1) and ERK2 play essential roles in osteoblast differentiation and in supporting osteoclastogenesis. Mol cell biol 2009; 29: 5843-5857.

26. Ge C, Xiao G, Jiang D, Franceschi RT. Critical role of the extracellular signal-regulated kinase-MAPK pathway in osteoblast differentiation and skeletal development. $J$ cell biol 2007; 176: 709-718.

27. Sandhu SK, Hampson G. The pathogenesis, diagnosis, investigation and management of osteoporosis. J clin pathol 2011; 64: 1042-1050.

28. Montagnani A. Bone anabolics in osteoporosis: Actuality and perspectives. World j orthoped 2014; 5: 247-254.

29. Provot S, Nachtrab G, Paruch J, Chen AP, Silva A, Kronenberg HM. A-raf and B-raf are dispensable for normal endochondral bone development, and parathyroid hormone-related peptide suppresses extracellular signal-regulated kinase activation in hypertrophic chondrocytes. Mol cell biol 2008; 28: 344-357.

30. Calvi LM, Sims NA, Hunzelman JL, Knight MC, Giovannetti A, Saxton JM et al. Activated parathyroid hormone/parathyroid hormone-related protein receptor in osteoblastic cells differentially affects cortical and trabecular bone. $J$ clin invest 2001; 107: 277-286.

31. Wein MN, Spatz J, Nishimori S, Doench J, Root D, Babij P et al. HDAC5 controls MEF2Cdriven sclerostin expression in osteocytes. J bone miner res 2015; 30: 400-411.

32. Bouxsein ML, Boyd SK, Christiansen BA, Guldberg RE, Jepsen KJ, Muller R. Guidelines for assessment of bone microstructure in rodents using micro-computed tomography. $J$ bone miner res 2010; 25: 1468-1486

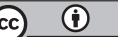

Cell Death and Disease is an open-access journal published by Nature Publishing Group. This work is licensed under a Creative Commons Attribution 4.0 International License. The images or other third party material in this article are included in the article's Creative Commons license, unless indicated otherwise in the credit line; if the material is not included under the Creative Commons license, users will need to obtain permission from the license holder to reproduce the material. To view a copy of this license, visit http://creativecommons.org/licenses/by/4.0/

(C) The Author(s) 2016

Supplementary Information accompanies the paper on Cell Death and Disease website (http://www.nature.com/cddis) 